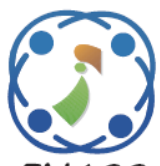

\title{
Estimation of Photovoltaic Cells Parameters Using Chaos Embedded Salp Swarm Algorithm
}

\author{
Dallel Nasri ${ }^{1}$ \\ Diab Mokeddem ${ }^{1 *}$ \\ Bachir Bourouba ${ }^{1}$ \\ ${ }^{1}$ Automatic laboratory of Setif, Department of Electrical Engineering, Faculty of Technology, \\ University of Ferhat Abbas Setif-1, Setif, 19000, Algeria \\ * Corresponding author's Email: mokeddem_d@yahoo.fr
}

\begin{abstract}
Solar photovoltaic (PV) systems have recently attracted researcher's attention as a clean source of energy. Thus, the importance to design appropriately the photovoltaic cells highly raises. The main problems faced in the design process are first, the development of a useful model describing the characteristics of the current vs. voltage able to simulate the real solar cells behaviours and then, the precise estimation of photovoltaic cells parameter values. This paper employs an improved version of Salp Swarm Algorithm called Chaotic Salp Swarm Algorithm (CSSA) for the parameters estimation of solar cells in both single and double diode models. CSSA approach benefits from chaotic maps proprieties, and has the advantage of providing good equilibrium between exploration and exploitation mechanisms as well. Performance of the proposed CSSA is compared to fourteen known algorithms. Experimental results demonstrate that the proposed algorithm has the ability to find the optimal solutions with an accurate estimation of parameters for the courant vs voltage characteristics of real solar cell with high performance.
\end{abstract}

Keywords: Photovoltaic, Estimation, Salp swarm algorithm, Chaotic.

\section{Introduction}

Nowadays, the need for a clean and renewable energy is the most interesting challenge for different countries all over the world. Regenerative energy is the solution key for a variety of serious problems as environmental pollution, global warming and fuel exhaustion. Hence, solar energy has urged as the most promising alternative, being available, nonexpandable and eco-friendly. In this regard, Photovoltaic (PV) systems are used to regenerate electrical energy starting from solar energy. Therefore, the modelling of solar cells is very important and consists of two steps: the formulation of the appropriate mathematical model and the precise estimation of the parameter values of the cell. For the mathematical model, the behaviour of a solar cell is governed by the current vs. the voltage characteristics. There exist two equivalent electronic circuits modelling the behaviour of a solar cell, for one is the single diode (SD) model and for another is the double diode (DD) model. Whatever the model selected, it is necessary to estimate all its parameters, and then identify their optimal values applied to the selected models, to approximate the experimental data obtained by the true solar cell.

In order to simulate, manage and optimize the real solar systems, many optimization techniques dealt with the identification of PV cell parameters. Guaranteed convergence particle swarm optimization (GCPSO) [1], enhanced leader particle swarm optimization (ELPSO) [2], improved JAYA (IJAYA) [3], artificial bee colony (ABC) [4], particle swarm optimization (PSO) [5, 6], genetic algorithms (GA) [7-9], evaporation rate based water cycle algorithm (ER-WCA) [10], simulated annealing (SA) [7], harmony search (HS) [11], teaching-learning based optimization (TBLO) [12], Bacterial Foraging Algorithm (BFA) [13], imperialist competitive algorithm (ICA) [14], self-adaptive teaching learning based optimization (SATLBO) [15], bird matting optimization (BMO) [16] and salp swarm algorithm.

The recently developed Salp swarm algorithm SSA [17] has proved its potential among population 
based metaheuristic methods due to simplicity and scalability. However, SSA still have the disadvantages of slow convergence speed and stagnation in local optima. To overcome these drawbacks and enhance the performance of standard SSA it was incorporated with chaos theory. In this way, feature selection using salp swarm algorithm with four chaotic maps was introduced in [18], where chaotic maps substitute random variables. In addition, Sayed et al. [19] proposed chaotic salp swarm algorithm using ten chaotic maps for solving benchmark dataset. Majhi et al. [20] uses Chaotic salp swarm algorithm for SDN multi-controller networks. Another chaotic salp swarm algorithm based on quadratic integrate and fire neural model for function optimization was presented by Ateya et al [21].

In this paper, Chaotic Salp Swarm Algorithm (CSSA) is employed for the first time to estimate the parameters of solar cells in both single and double diode models. In CSSA the logistic map is used to replace the random parameter in the mathematical model of the original SSA to provide a good balance between local and global searches.

The proposed CSSA is compared with recent well-known algorithms for the considered parameter extraction problem as chaotic teaching-learning algorithm (CTLA) [22], biogeography-based learning particle swarm optimization (BLPSO) [23], simulated annealing (SA) [7], competitive swarm optimizer (CSO) [24], levy flight trajectory-based whale optimization algorithm (LWOA) [25], memetic adaptive differential evolution (MADE) [26], cuckoo search algorithm (CS) [27], hybridizing cuckoo search algorithm with biogeography-based optimization (CS-BBO) [27], teaching-learningbased artificial bee colony (TLBO-ABC) [28], multiple learning backtracking search algorithm (LBSA) [29], chaotic whale optimization algorithm (CWOA) [30], improved opposition-based sine cosine algorithm (ISCA) [31], hybrid particle swarm optimization with whale optimization algorithm (PSO-WOA) [32], hybrid algorithm based on grey wolf optimizer and cuckoo search (GWOCS) [33].

Experimental results proved efficiency of the proposed approach to identify the PV cells parameters.

The rest of the paper is organised as follows: Section 2 presents the standard salp swarm algorithm. Then, Section 3 detailed the chaotic salp swarm algorithm. The photovoltaic models and optimization problem formulation are presented in sections 4 and 5 respectively. Simulation and comparison results are discussed in section 6. Finally, section 7 summarizes the conclusion.

\section{Standard salp swarm algorithm}

The Salp Swarm Algorithm (SSA) is a recent nature-inspired metaheuristic optimizer proposed by et al. [17] in 2017. The SSA algorithm mimics the swarming behaviour of salps that live in groups by forming a salp chain. The first salp is denoted as the leader, while the other salps are followers. In the mathematical model of the SSA, location of the leader salp should be updated following Eq. (1).

$$
x_{j}^{1}=\left\{\begin{array}{l}
F_{j}+c_{1}\left(\left(u b_{j}-l b_{j}\right) c_{2}+l b_{j}\right) c_{3} \geq 0.5 \\
F_{j}-c_{1}\left(\left(u b_{j}-l b_{j}\right) c_{2}+l b_{j}\right) c_{3}<0.5
\end{array}\right.
$$

where $x_{j}^{1}, j$ shows the position of the first salp (leader) in the $j$ th dimension, $F_{j}$ is the position of the food source in the $j$ th dimension, $u b_{j}$ indicates the upper bound of $j$ th dimension, $l b_{j}$ indicates the lower bound of $j$ th dimension, $c_{1}, c_{2}$, and $c_{3}$ are random numbers.

Eq. (1) shows that the leader only updates its position with respect to the food source. The coefficient $c_{1}$ is the most important parameter in SSA algorithm, because it balances exploration and exploitation and can be defined by:

$$
c_{1}=2 e^{-\left(\frac{4 k}{L}\right)^{2}}
$$

where $k$ represents the current iteration, $L$ indicates the maximum number of iterations.

The parameter $c_{2}$ and $c_{3}$ are random numbers uniformly generated in the interval of $[0,1]$. In fact, they dictate if the next position in $j$ th dimension should be towards positive infinity or negative infinity as well as the step size. (3):

The followers' positions are updated using Eq.

$$
x_{j}^{i}=\frac{1}{2}\left(x_{j}^{i}+x_{j}^{i-1}\right) \quad i \geq 2
$$

where $x_{j}^{i}$ represents the position of the $i$ th follower at $j$ th dimension.

The pseudocode of the SSA algorithm is presented in Algorithm 1.

\section{Chaotic salp swarm algorithm}

Although SSA algorithm is among the most performing techniques, it still suffers from slow convergence speed and stagnation into local optimum. So, to reduce these disadvantages and improve the algorithm's efficiency, we introduced chaos to SSA algorithm. Chaos theory studies the behaviour of 
Algorithm 1. Pseudo code of SSA algorithm

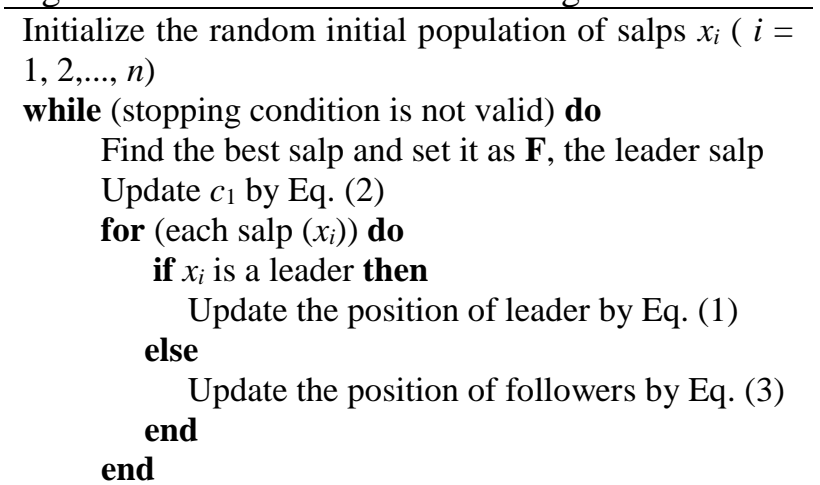

Update all salps based on the upper and lower bounds of variables

Return F

systems that follow deterministic laws but appear random and unpredictable. In other words, a chaotic system is a dynamical system that has a sensitive dependence on its initial conditions; small changes in those conditions can lead to quite different outcomes [18-20]. Due to the ergodicity, non-repetition and sensitivity dependence on initial conditions properties of chaos, the algorithm can perform overall search steps at higher speeds compared to the stochastic searches relying on probabilities. Thus, it is very beneficial to replace randomness in metaheuristic algorithms by chaotic maps to ensure that solutions generated by the algorithm can be diverse enough to potentially reach the global optimum in the search space and avoid stagnation into local optimum [34].

Chaotic sequences were successfully applied in a number of popular nature inspired metaheuristic algorithms as: krill herd optimization algorithm [35], grey wolf optimization algorithm [36], grasshopper optimization algorithm [37], firefly algorithm (FA) [38], bee colony algorithm [39], whale optimization algorithm [40] and bat algorithm [41].

In SSA algorithm c2 parameter which contain random numbers can be modified with chaos mappings. Our suggestion would be using the successions made by the logistic mapping instead of above-mentioned random numbers. Sequences generated by the logistic mapping are formulated below:

$$
x(k+1)=4 x(k)(1-x(k))
$$

Where $x(k)$ is the $k$ th chaotic number, with $k$ denoting the iteration number. Obviously, $x \in[0,1]$ and that $x(0)$ should not be $0,0.25,0.5,0.75$ or 1 . Fig. 1 shows the chaotic $x(t)$ value using a logistic map for 100 iterations where $x(0)=0.7$.

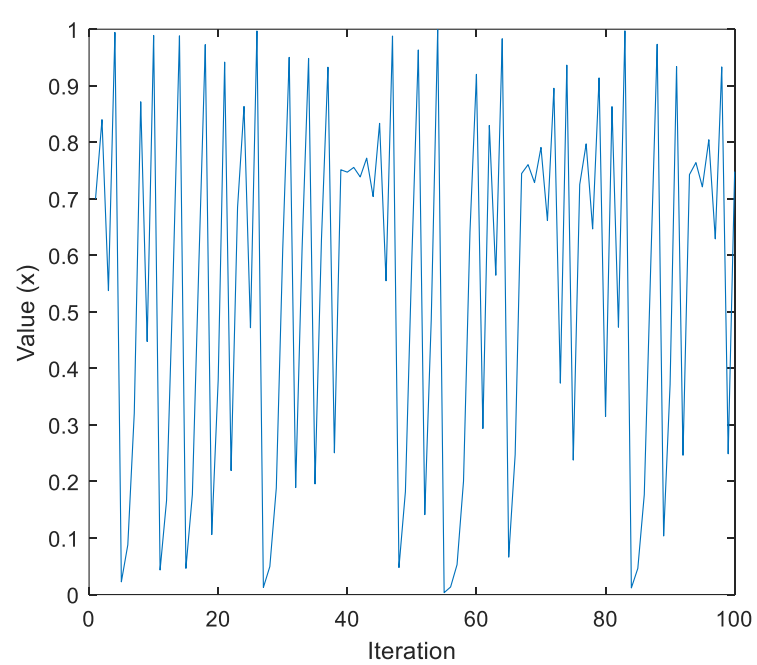

Figure. 1 Chaotic values distribution's during 100 iteration

\section{Photovoltaic models}

In general, there exist two models of solar cells: single diode (SD) and double diode (DD) [30]. A good mathematical model is necessary to generate a precise design of solar cells with an accurate parameter values estimation. This section describes the SD and DD models and formulate their objective functions.

\subsection{Double diode model (DD)}

As its name implies, a double diode model consists of two diodes: One of these diodes is set as a rectifier and the second diode is used to model the charge recombination current and some nonidealities. These diodes are used to shunt the photogenerated current source $\left(I_{p h}\right)$ and are connected in series with a resistor [5, 19]. Fig. 2 defines the equivalent circuit for a double diode (DD) model. The cell terminal current is computed as follows:

$$
I_{t}=I_{p h}-I_{d 1}-I_{d 2}-I_{s h}
$$

where the terminal current is $I_{t}, I_{p h}$ is the photogenerated current, $I_{d 1}, I_{d 2}$ are the first and second diode currents respectively, whereas $I_{s h}$ is the shunt resistor current.

For the proper modelling of PV cells, we have used the Shockley diode equation. Hence, Eq. (5) is rewritten as shown in Eq. (6).

$$
\begin{gathered}
I_{t e}=I_{p h-} I_{s d 1}\left[\exp \left(\left(\frac{V_{d}}{n_{1} \cdot V_{t e}}\right)-1\right]\right. \\
-I_{s d 2}\left[\exp \left(\left(\frac{V_{d}}{n_{2} \cdot V_{t e}}\right)-1\right]-\frac{V_{t}+I_{t e} \cdot R_{s}}{R_{s h}}\right.
\end{gathered}
$$




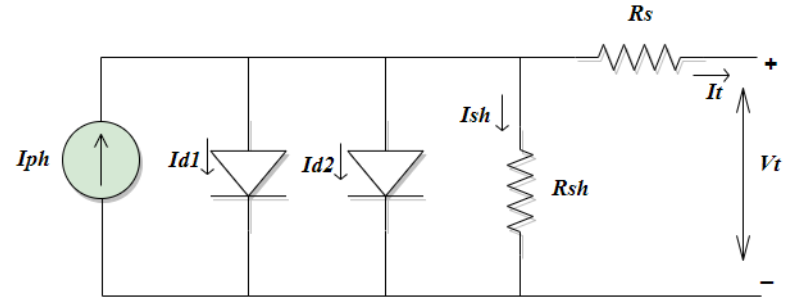

Figure. 2 Solar cells with double diode model

where $V_{t}$ is the terminal voltage, whereas the series and shunt resistances are represented by $R_{s}$ and $R_{s h}$ respectively. $I_{s d 1}$ and $I_{s d 2}$ are the diffusion and saturation current, respectively. $n_{1}$ and $n_{2}$ are respectively the diffusion and recombination diode ideality factors, Therefore, $\left(R_{s}, R_{s h}, I_{p h}, I_{s d 1}, I_{s d 2}, n_{1}\right.$ and $\left.n_{2}\right)$ are the seven unknown parameters in Eq. (6). The identification of these parameters improves the optimal performance of the solar cell.

\subsection{Single diode model (SD)}

The diffusion current $I_{s d 1}$ and the saturation current $I_{s d 2}$ currents in a single diode model are represented by a non-physical ideality factor $n$. The equivalent circuit of single diode model is presented in Fig. 3. In this model, only five parameters are to be estimated $R_{s}, R_{s h}, I_{p h}, I_{s d}$ and $n$. Then, the single diode model adapted is given in Eq. (7):

$$
I_{t e}=I_{p h-} I_{s d}\left[\exp \left(\left(\frac{V_{t}+I_{t e} \cdot R_{S}}{n \cdot V_{t}}\right)-1\right]-\frac{V_{t}+I_{t e} \cdot R_{S}}{R_{s h}}\right.
$$

\section{Optimization problem formulation}

To define the optimization problem, the parameters of each equivalent circuit (DD and SD) must be formulated as variable vectors. The bounds of the parameters were chosen in order to include RTC France PV cell technology, in conformity with the literature. Eq. (8) indicates the ranges for both single and double diode models.

$$
\min f(x), x=\left[x_{1}, x_{2} \ldots . x_{d}\right], d \in[5,7]
$$

with

$$
\begin{array}{cc}
d=5(S D) & d=7(D D) \\
0 \leq x_{1}\left(R_{s}\right) \leq 0.5 & 0 \leq x_{1}\left(R_{s}\right) \leq 0.5 \\
0 \leq x_{2}\left(R_{s h}\right) \leq 100 & 0 \leq x_{2}\left(R_{s h}\right) \leq 100 \\
0 \leq x_{3}\left(I_{p h}\right) \leq 1 & 0 \leq x_{3}\left(I_{p h}\right) \leq 1 \\
0 \leq x_{4}\left(I_{0}\right) \leq 1 & 0 \leq x_{4}\left(I_{s d 1}\right) \leq 1 \\
1 \leq x_{5}(n) \leq 2 & 0 \leq x_{5}\left(I_{s d 2}\right) \leq 1 \\
& 1 \leq x_{6}\left(n_{1}\right) \leq 2 \\
& 1 \leq x_{7}\left(n_{2}\right) \leq 2
\end{array}
$$

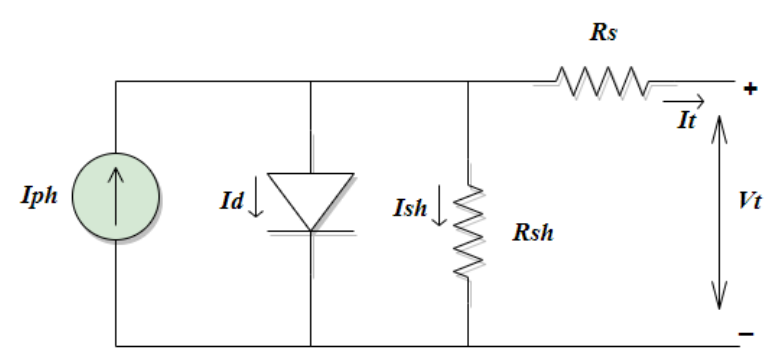

Figure. 3 Solar cells with single diode model

To determine the quality of the estimated parameters we define the objective function of the Root Mean Square Error (RMSE):

$$
\operatorname{Min} \operatorname{RMSE}(x)=\operatorname{Min} \sqrt{\frac{1}{N} \sum_{i=1}^{N}\left(I_{t}-I_{t e}\right)^{2}}
$$

where $N$ represents a set of empirical point $\left(I_{t}, V_{t}\right)$ measured and $I_{t e}$ is the estimated value of current as a function of the unknown parameters $x$ that characterize the model described by Eqs. (6) and (7). A good set of parameters produces a precise approximation between the measurements of the current with respect to the voltage $(\mathrm{I}-\mathrm{V})$ of the physical cell and the values of the mathematical model [30]. In the case of a solar cell with a single diode the error is defined by Eq. (9):

$$
\begin{gathered}
f_{S D}\left(V_{t}, I_{t}, x\right)=I_{t}-x_{3}+x_{4} \\
{\left[\exp \left(\frac{q\left(V_{t}+x_{1} \cdot I_{t e}\right)}{x_{5} \cdot k \cdot T}\right)-1\right]+\frac{V_{t+} x_{1} \cdot I_{t e}}{x_{2}}}
\end{gathered}
$$

Meanwhile, the error in case of double diode is given by Eq. (10):

$$
\begin{array}{r}
f_{D D}\left(V_{t} I_{t}, x\right)=I_{t}-x_{3}+x_{4}\left[\exp \left(\frac{q\left(V_{t}+x_{1} \cdot I_{t e}\right.}{x_{6} \cdot k \cdot T}\right)\right] \\
+x_{5}\left[\exp \left(\frac{q\left(V_{t}+x_{1} \cdot I_{t e}\right.}{x_{7} \cdot k \cdot T}\right)-1\right]+\frac{-1}{x_{t}+x_{1} \cdot I_{t e}}
\end{array}
$$

In both functions $\left(f_{D D}\right.$ and $\left.f_{S D}\right)$, the values of $V_{t}$ and $I t$ are experimentally collected from the solar cell, $x$ is a vector that contains the model parameters, where $x=\left\{R_{s}, R_{s h}, I_{p h}, I_{s d 1}, I_{s d 2}, n_{1}, n_{2}\right\}$ is the model parameters for DD and $x=\left\{R_{s}, R_{s h}, I_{p h}, I_{s d}, n\right\}$ for SD. In the Shockley diode equation, $q=1.602 \times 10^{-19}$ (coulombs) is the magnitude of charge on an electron, $k=1.381 \times 10^{-23}(\mathrm{~J} / \mathrm{K})$ is the Boltzmann constant and $T$ is the cell temperature $(\mathrm{K})$. 


\section{Results and discussion}

\subsection{Comparison of CSSA and SSA}

In this section, we aim to apply the Chaotic Salp Swarm Algorithm (CSSA), to estimate the parameters of solar cell models. Then, we evaluate the performance of the proposed CSSA compared to the standard SSA. For a fair comparison, CSSA and SSA algorithms are executed 10 times under the same conditions of 30 search agents and 500 iterations. The experimental measurements adopted in this study are taken from [30], where authors uses a silicon solar cell with a diameter of $57 \mathrm{~mm}$ to measure current and voltage of 26 samples under the conditions: one sun $\left(1000 \mathrm{~W} / \mathrm{m}^{2}\right)$ at $\mathrm{T}=33^{\circ} \mathrm{C}$ and respecting the constraints defined by Eq. (8).

The parameters of the identified photovoltaic cells obtained by the methods of CSSA and SSA using Root Mean Square Error (RMSE) for the single diode (SD) and double diode (DD) models are shown in Tables 1 and 2 respectively

From Tables 1 and 2, we observe that the algorithm CSSA with the RMSE performance criterion has a better performance (smaller value) to identify the parameters of both SD and DD models of solar compared to the standard SSA obtained values. Fig. 4 shows the evolution of the objective function (RMSE) by the two competitive algorithms CSSA and SSA in accordance to the number of iterations in both cases of single and double diode models. This figure shows that the CSSA algorithm has a faster convergence speed compared to standard SSA algorithm.

Table 1. Comparison results for the SD model

\begin{tabular}{|c|c|c|}
\hline Parameters & CSSA & SSA \\
\hline$I_{p h}(A)$ & 0.755081 & 0.729161 \\
\hline$I_{s d}(\mu A)$ & 0226751 & 0.332893 \\
\hline$n$ & 1.44753 & 1.49995 \\
\hline$R_{s}(\Omega)$ & 0.0351985 & 0.0308495 \\
\hline$R_{s h}(\Omega)$ & 52.4437 & 53.1931 \\
\hline $\mathrm{RMSE}$ & $8.9064 \times 10^{-3}$ & 0.010368 \\
\hline
\end{tabular}

Table 2. Comparison results for the DD model

\begin{tabular}{|c|c|c|}
\hline Parameters & CSSA & SSA \\
\hline$I_{p h}(A)$ & 0.761222 & 0.758726 \\
\hline$I_{s 1}(\mu A)$ & 0.216042 & 0.107877 \\
\hline$I_{s 2}(\mu A)$ & 0.262701 & 0.578480 \\
\hline$n_{1}$ & 1.74375 & 1.38726 \\
\hline$n_{2}$ & 1.46755 & 1.82912 \\
\hline$R_{s}(\Omega)$ & 0.0362049 & 0.0390374 \\
\hline$R_{s h}(\Omega)$ & 51.1869 & 89.3002 \\
\hline $\mathrm{RMSE}$ & $1.0501 \times 10^{-3}$ & $2.07 \times 10^{-3}$ \\
\hline
\end{tabular}

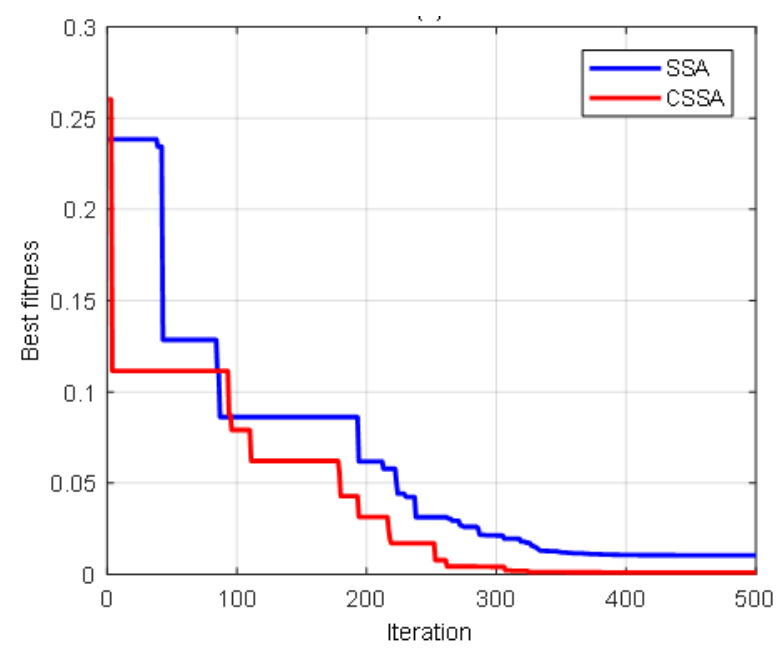

(a)

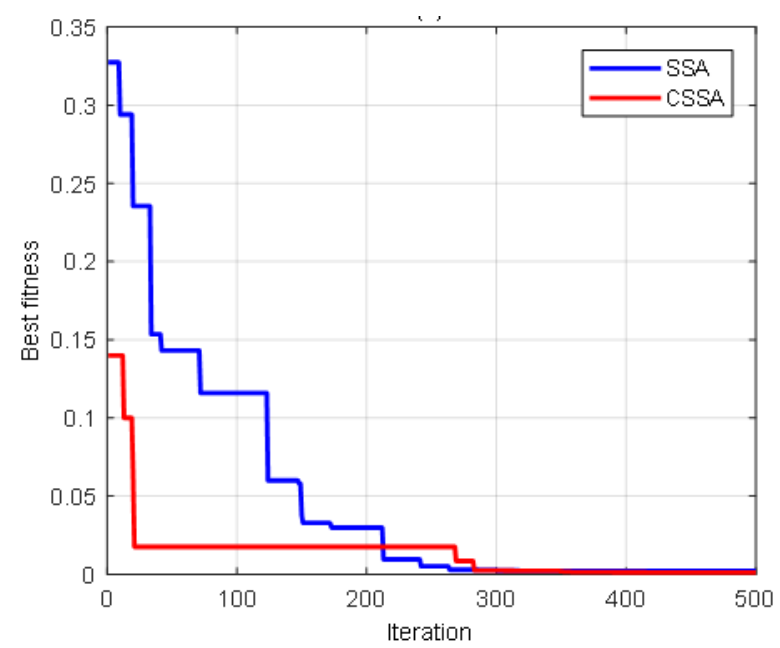

(b)

Figure. 4 RMSE evolution: (a) single diode model and (b) double diode model obtained by CSSA and SSA

Fig. 5 shows the current vs the voltage and the power vs the voltage curves for SD model. These curves are calculated using the parameters estimated by CSSA and SSA algorithms and the experimental data.

From Fig. 5 we can clearly notice that, the solutions yielded by the proposed CSSA algorithm for the solar cell with single diode model can accurately represent the characteristics of the real photovoltaic cell than the results obtained by SSA algorithm. It also indicates that CSSA algorithm obtained an accurate approximation to the current estimated.

The current vs the voltage and the power vs the voltage characteristic curves for DD model are shown in Fig. 6. These curves are reconstructed using the parameters estimated by CSSA and SSA algorithms and the experimental data. Analyzing Fig. 6, we can see that CSSA algorithm has the potential to provide 


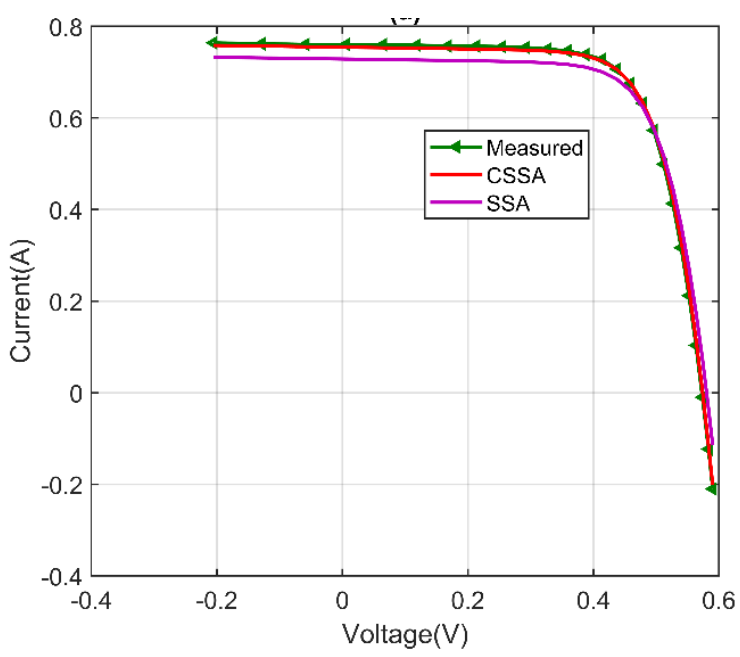

(a)

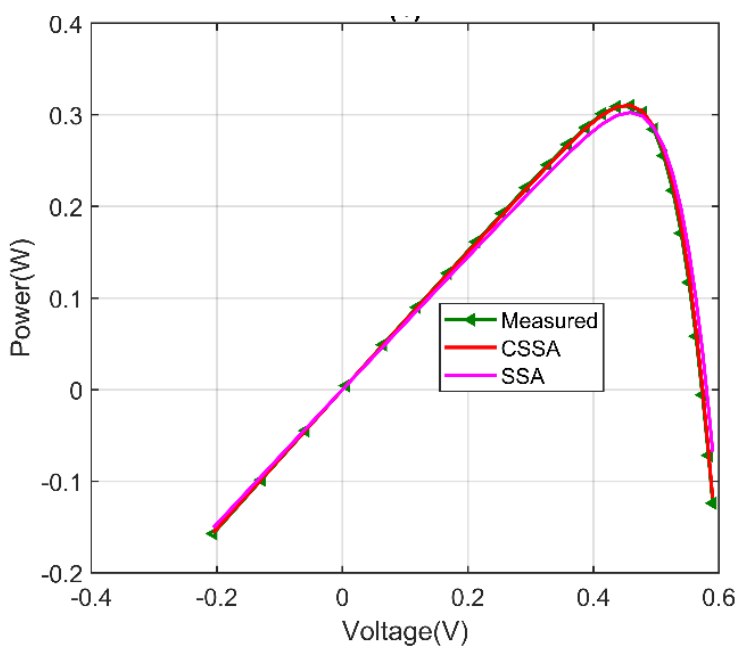

(b)

Figure. 5 :(a) Current vs measured voltage and (b) power vs the measured voltage for the SD model computed using CSSA and SSA algorithms

results that accurately approximate the experimentally measured data.

To further evaluate the quality of values obtained by the proposed CSSA algorithm for the various parameters; the current estimated with these values is compared with the experimental current. For this, the individual absolute error (IAE) for each of the 26 curve points of the measured current is calculated using Eq. (11).

$$
I A E=\left|I_{t}-I_{t e}\right|
$$

Fig. 7 shows the individual absolute error IAE between the experimentally measured current and that estimated for the single diode model. Analyzing the plot of Fig. 7, we can clearly notice that all IAE values are less than 0.014 , which indicate a very close approximation of the estimated current values to the measured current values.

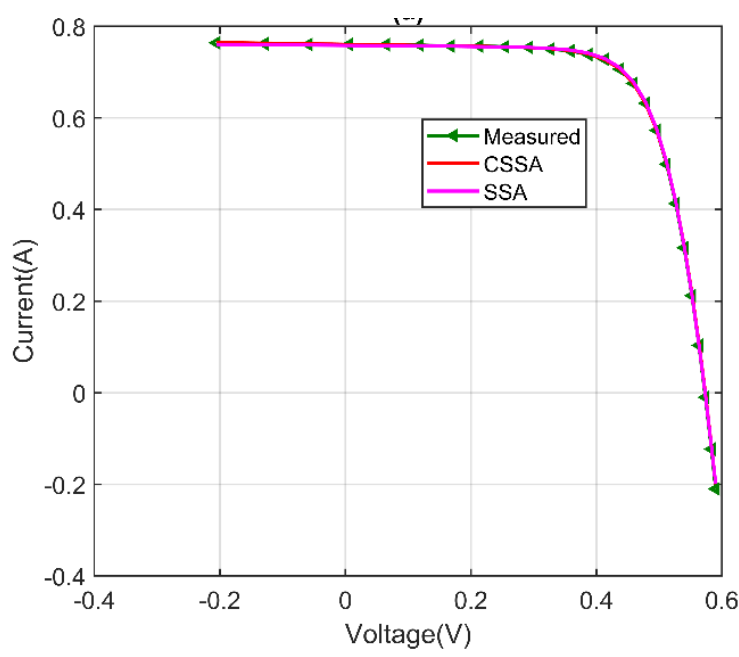

(a)

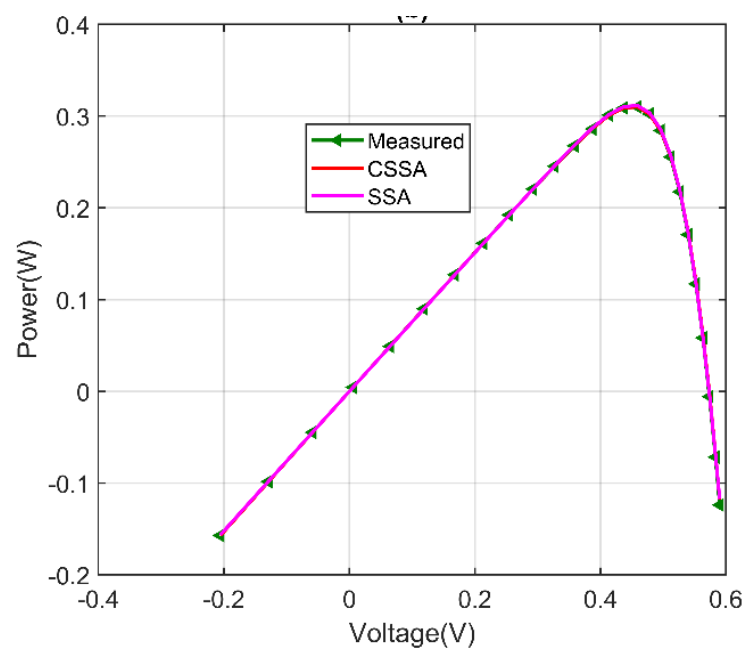

(b)

Figure. 6 :(a) Current vs measured voltage and (b) power vs the measured voltage for the DD model computed using CSSA and SSA algorithms

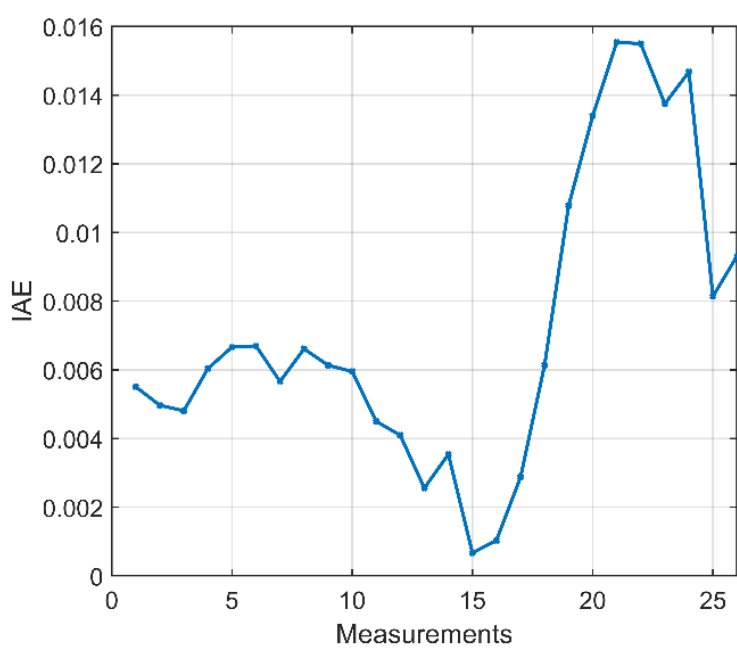

Figure. 7 Individual absolute error curve between experimentally measured and estimated currents for single diode model 


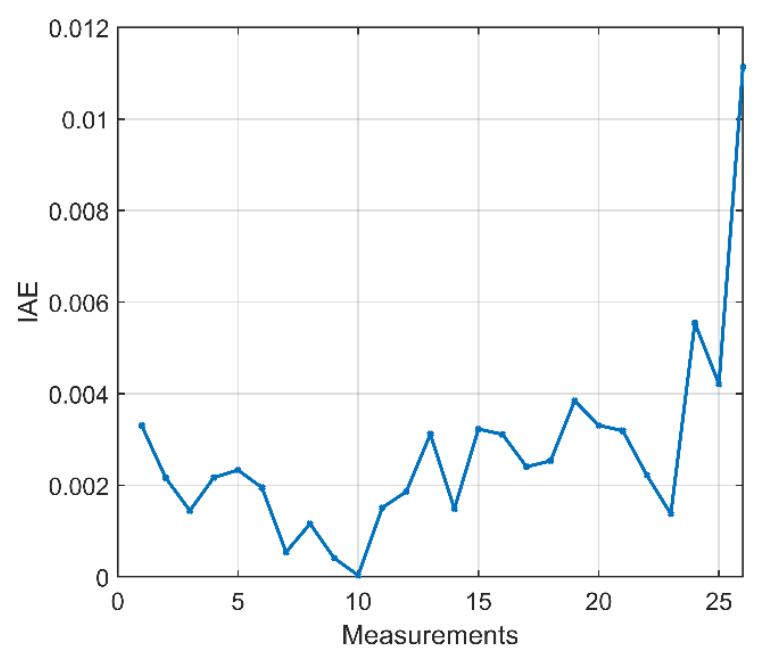

Figure. 8 Individual absolute error curve between experimentally measured and estimated currents for double diode model.

In case of double diode model, the individual absolute error IAE curve between the estimated and experimentally measured currents is shown in Fig. 8. All the IAE values obtained are less than 0.008, indicating that the estimated current and the measured current curves are coincident.

From the individual absolute error (IAE) curves of experimentally measured and estimated currents for both SD and DD models, we can conclude that the proposed CSSA algorithm has the potential to estimate very precise parameters values for the solar cell.

\subsection{Comparison of CSSA with well-known algorithms}

To evaluate the performance of CSSA algorithm to identify the parameters of PV cell using SD and DD models, a fair comparison with a set of optimization algorithms is conducted in this subsection. The comparative algorithms considered are chaotic teaching-learning algorithm (CTLA) [22], biogeography-based learning particle swarm optimization (BLPSO) [23], simulated annealing (SA) [7], competitive swarm optimizer (CSO) [24], levy flight trajectory-based whale optimization algorithm (LWOA) [25], memetic adaptive differential evolution (MADE) [26] cuckoo search algorithm (CS) [27], hybridizing cuckoo search algorithm with biogeography-based optimization (CS-BBO) [27], teaching-learning- based artificial bee colony (TLBO-ABC) [28], multiple learning backtracking search algorithm (LBSA) [29], chaotic whale optimization algorithm (CWOA) [30], improved opposition-based sine cosine algorithm (ISCA) [31], hybrid particle swarm optimization with whale optimization algorithm (PSO-WOA) [32], hybrid algorithm based on grey wolf optimizer and cuckoo search (GWOCS) [33] and the proposed CSSA.

Tables 3 and 4 report the optimal estimated parameters obtained by all the competitive algorithms for the SD and DD models respectively. Comparing the results in Table 3 for the single diode model, CSSA outperforms CTLA, BLPSO, SA, CSO, LWOA, CS, LBSA, PSO-WOA algorithms obtaining the lowest RMSE value of $9.906 \times 10^{-4}$ and showed quite competitive performance with the second best RMSE value after MADE, CS-BBO, TLBO-ABC, CWOA, ISCA and GWOCS.

Analysing the results of Table 4 for the double diode model, CSSA showed the best performance over CTLA, BLPSO, SA, CSO, LWOA, CS, PSOWOA algorithms achieving an RMSE value of $1.050 \times 10^{-3}$ and not much outperformed by MADE, CS-BBO, TLBO-ABC, LBSA, CWOA, ISCA and GWOCS.

Table 3. Comparison of estimated parameters by the applied algorithms for the SD model

\begin{tabular}{|c|c|c|c|c|c|c|}
\hline Algorithm & $R_{s}(\Omega)$ & $R_{s h}(\Omega)$ & $I_{p h}(A)$ & $I_{s d}(\mu \mathrm{A})$ & $n$ & RMSE \\
\hline CTLA & 0.0357 & 61.1131 & 0.7650 & 0.4280 & 1.5092 & $1.09 \times 10^{-3}$ \\
\hline BLPSO & 0.0347 & 96.5115 & 0.7599 & 0.4977 & 1.5257 & $1.48 \times 10^{-3}$ \\
\hline SA & 0.0345 & 43.1034 & 0.7620 & 0.4798 & 1.5172 & $1.70 \times 10^{-3}$ \\
\hline CSO & 1.2122 & 1689.005 & 1.0205 & 0.3658 & 48.8206 & $1.63 \times 10^{-3}$ \\
\hline LWOA & 1.2218 & 1272.0197 & 1.0284 & 0.3145 & 48.2413 & $1.087 \times 10^{-3}$ \\
\hline MADE & 0.0364 & 53.7185 & 0.7608 & 0.3230 & 1.4812 & $9.8602 \times 10^{-4}$ \\
\hline CS & 0.03492 & 43.84232 & 0.76048 & 0.36015 & 1.4929 & $2.0119 \times 10^{-3}$ \\
\hline CS-BBO & 0.03638 & 53.71852 & 0.76078 & 0.32302 & 1.48118 & $9.8602 \times 10^{-4}$ \\
\hline TLBO-ABC & 0.03638 & 53.71636 & 0.76078 & 0.32302 & 1.48118 & $9.8602 \times 10^{-4}$ \\
\hline LBSA & 0.0362 & 59.0978 & 0.7606 & 0.34618 & 1.4881 & $1.0143 \times 10^{-3}$ \\
\hline CWOA & 0.03636 & 53.7987 & 0.76077 & 0.3239 & 1.4812 & $9.8602 \times 10^{-4}$ \\
\hline ISCA & 0.03638 & 53.7182 & 0.760778 & 0.323017 & 1.4812 & $9.8602 \times 10^{-4}$ \\
\hline PSO-WOA & 0.036124 & 59.323133 & 0.760563 & 0.340158 & 1.4863 & $1.0710 \times 10^{-3}$ \\
\hline GWOCS & 0.03639 & 53.6320 & 0.760773 & 0.32192 & 1.4808 & $9.8607 \times 10^{-4}$ \\
\hline CSSA & 0.03519 & 52.4437 & 0.755081 & 0226751 & 1.44753 & $8.9064 \times 10^{-3}$ \\
\hline
\end{tabular}


Table 4. Comparison of estimated parameters by the applied algorithms for the DD model

\begin{tabular}{|c|c|c|c|c|c|c|c|c|}
\hline Algorithm & $R_{s}(\Omega)$ & $R_{s h}(\Omega)$ & $I_{p h}(A)$ & $I_{s d l}(\mu \mathrm{A})$ & $I_{s d 2}(\mu \mathrm{A})$ & $n_{1}$ & $n_{2}$ & RMSE \\
\hline CTLA & 0.0313 & 89.6464 & 0.7570 & 0.8542 & 0.3812 & 1.7879 & 1.5230 & $1.32 \times 10^{-3}$ \\
\hline BLPSO & 0.0338 & 78.6922 & 0.7607 & 0.5481 & 0.0542 & 1.5442 & 1.5765 & $1.57 \times 10^{-3}$ \\
\hline SA & 0.0345 & 43.1034 & 0.7623 & 0.4767 & 0.0100 & 1.5172 & 2.0000 & $1.90 \times 10^{-2}$ \\
\hline CSO & 0.0409 & 15.773 & 0.7628 & 0.7954 & 0.6780 & 1.6936 & 1.8138 & $1.70 \times 10^{-3}$ \\
\hline LWOA & 0.0355 & 86.8763 & 0.7597 & 0.2342 & 0.3709 & 1.4679 & 1.6989 & $1.31 \times 10^{-3}$ \\
\hline MADE & 0.03680 & 55.4329 & 0.7608 & 0.7394 & 0.2246 & 1.9963 & 1.4505 & $9.8261 \times 10^{-4}$ \\
\hline CS & 0.03530 & 97.73242 & 0.76223 & 0.02732 & 0.50832 & 1.7027 & 1.52893 & $2.4440 \times 10^{-3}$ \\
\hline CS-BBO & 0.03674 & 55.48544 & 0.76078 & 0.74935 & 0.22597 & 2 & 1.45102 & $9.8249 \times 10^{-4}$ \\
\hline TLBO-ABC & 0.03667 & 54.66797 & 0.76081 & 0.42394 & 0.24011 & 1.9075 & 1.45671 & $9.8415 \times 10^{-4}$ \\
\hline LBSA & 0.0363 & 60.1880 & 0.7606 & 0.29814 & 0.27096 & 1.4760 & 1.9202 & $1.0165 \times 10^{-3}$ \\
\hline CWOA & 0.03666 & 55.2016 & 0.76077 & 0.24150 & 0.6 & 1.4565 & 1.9899 & $9.8272 \times 10^{-4}$ \\
\hline ISCA & 0.03674 & 55.48543 & 0.76078 & 0.74935 & 0.22597 & 2 & 1.45102 & $9.8237 \times 10^{-4}$ \\
\hline PSO-WOA & 0.034223 & 82.82299 & 0.761091 & 0.20123 & 0.93611 & 1.4633 & 1.773674 & $1.6700 \times 10^{-3}$ \\
\hline GWOCS & 0.03666 & 54.7331 & 0.76076 & 0.53772 & 0.24855 & 2 & 1.4588 & $9.8334 \times 10^{-4}$ \\
\hline CSSA & 0.0362 & 51.1869 & 0.7612 & 0.2160 & 0.2627 & 1.7437 & 1.4675 & $1.050 \times 10^{-3}$ \\
\hline
\end{tabular}

The proposed CSSA with singer map showed a good performance for parameter estimation of both single and double diode models, due to good equilibrium between exploration and exploitation mechanisms provided by embedding chaos to standard SSA.

\section{Conclusion}

This paper applied an optimization method based on metaheuristic algorithm and chaotic maps called Chaotic Salp Swarm Algorithm (CSSA) for estimating the parameters of a single diode (SD) and double diode (DD) models of a solar cell. In this study, the logistic map is integrated into the original Salp Swarm algorithm (SSA), to enhance its convergence speed and overall performance, by substituting the algorithm's random parameter. Performance of the proposed CSSA is compared with CTLA, BLPSO, SA, CSO, LWOA, MADE, CS, CSBBO, TLBO-ABC, LBSA, CWOA, ISCA, PSOWOA and GWOCS algorithms based on the minimization of the objective function of root mean squared error.

Experimental results proved evidently good performance of CSSA over other comparative algorithms for parameter estimation problem in both SD and DD models. In future work, we will extend the use of CSSA to other challenging renewable energy problems.

\section{Conflicts of Interest}

The authors declare no conflict of interest.

\section{Author Contributions}

Conceptualization, methodology, software, validation, writing, investigation, Dallel Nasri; formal analysis, data curation, review and editing, supervision, Diab Mokeddem; supervision, Bachir Bourouba.

\section{References}

[1] H. G. G. Nunes, J. A. N. Pombo, S. Mariano, M. R. A. Calado, and J. A. M. F. De Souza, "A new high performance method for determining the parameters of PV cells and modules based on guaranteed convergence particle swarm optimization", Applied Energy, Vol. 211, pp. 774-791, 2018.

[2] A. R. Jordehi, "Enhanced leader particle swarm optimisation (ELPSO): an efficient algorithm for parameter estimation of photovoltaic (PV) cells and modules", Solar Energy, Vol. 159, pp. 78-87, 2018.

[3] K. Yu, J. J. Liang, B. Y. Qu, X. Chen, and H. Wang, "Parameters identification of photovoltaic models using an improved JAYA optimization algorithm", Energy Conversion and Management, Vol. 150, pp. 742-753, 2017.

[4] D. Oliva, E. Cuevas, and G. Pajares, "Parameter identification of solar cells using artificial bee colony optimization", Energy, Vol. 72, pp. 93102, 2014.

[5] M. Ye, X. Wang, and Y. Xu, "Parameter extraction of solar cells using particle swarm optimization", Journal of Applied Physics, Vol. 105, No. 9, pp. 94502, 2009.

[6] H. Wei, J. Cong, X. Lingyun, and S. Deyun, "Extracting solar cell model parameters based on chaos particle swarm algorithm", In Proc. of 2011 International Conf. on Electric Information and Control Engineering, pp. 398402, 2011. 
[7] K. M. El-Naggar, M. R. AlRashidi, M. F. AlHajri, and A. K. Al-Othman, "Simulated annealing algorithm for photovoltaic parameters identification", Solar Energy, Vol. 86, No. 1, pp. 266-274, 2012.

[8] J. A. Jervase, H. Bourdoucen, and A. Al-Lawati, "Solar cell parameter extraction using genetic algorithms", Measurement Science and Technology, Vol. 12, No. 11, pp. 1922, 2001.

[9] J. Appelbaum and A. Peled, "Parameters extraction of solar cells-A comparative examination of three methods", Solar Energy Materials and Solar Cells, Vol. 122, pp. 164173, 2014.

[10] D. Kler, P. Sharma, A. Banerjee, K. P. S. Rana, and V. Kumar, "PV cell and module efficient parameters estimation using Evaporation Rate based Water Cycle Algorithm", Swarm and Evolutionary Computation, Vol. 35, pp. 93-110, 2017.

[11] A. Askarzadeh and A. Rezazadeh, "Parameter identification for solar cell models using harmony search-based algorithms", Solar Energy, Vol. 86, No. 11, pp. 3241-3249, 2012.

[12] Q. Niu, H. Zhang, and K. Li, "An improved TLBO with elite strategy for parameters identification of PEM fuel cell and solar cell models", International Journal of Hydrogen Energy, Vol. 39, No. 8, pp. 3837-3854, 2014.

[13] N. Rajasekar, N. K. Kumar, and R. Venugopalan, "Bacterial foraging algorithm based solar PV parameter estimation", Solar Energy, Vol. 97, pp. 255-265, 2013.

[14] A. Fathy and H. Rezk, "Parameter estimation of photovoltaic system using imperialist competitive algorithm", Renewable Energy, Vol. 111, pp. 307-320, 2017.

[15] K. Yu, X. Chen, X. Wang, and Z. Wang, "Parameters identification of photovoltaic models using self-adaptive teaching-learningbased optimization", Energy Conversion and Management, Vol. 145, pp. 233-246, 2017.

[16] A. Askarzadeh and A. Rezazadeh, "Extraction of maximum power point in solar cells using bird mating optimizer-based parameters identification approach", Solar Energy, Vol. 90, pp. 123-133, 2013.

[17] S. Mirjalili, A. H. Gandomi, S. Z. Mirjalili, S. Saremi, H. Faris, and S. M. Mirjalili, "Salp Swarm Algorithm: A bio-inspired optimizer for engineering design problems", Advances in Engineering Software, Vol. 114, pp. 163-191, 2017.

[18] S. Ahmed, M. Mafarja, H. Faris, and I. Aljarah, "Feature selection using salp swarm algorithm with chaos", in Proc. of the 2nd International Conf. on Intelligent Systems, Metaheuristics \& Swarm Intelligence, pp. 65-69, 2018.

[19] G. I. Sayed, G. Khoriba, and M. H. Haggag, "A novel chaotic salp swarm algorithm for global optimization and feature selection", Applied Intelligence, Vol. 48, No. 10, pp. 3462-3481, 2018.

[20] S. K. Majhi, A. Mishra, and R. Pradhan, "A chaotic salp swarm algorithm based on quadratic integrate and fire neural model for function optimization," Progress in Artificial Intelligence, Vol. 8, No. 3, pp. 343-358, 2019.

[21] A. A. Ateya, A. Muthanna, A. Vybornova, A. D. Algarni, A. Abuarqoub, Y. Koucheryavy, A. Koucheryavy, "Chaotic salp swarm algorithm for SDN multi-controller networks", Engineering Science and Technology, an International Journal, Vol. 22, No. 4, pp. 10011012, 2019.

[22] A. Farah, T. Guesmi, H. H. Abdallah, and A. Ouali, "A novel chaotic teaching-learningbased optimization algorithm for multi-machine power system stabilizers design problem", International Journal of Electrical Power \& Energy Systems, Vol. 77, pp. 197-209, 2016.

[23] X. Chen, H. Tianfield, C. Mei, W. Du, and G. Liu, "Biogeography-based learning particle swarm optimization", Soft Computing, Vol. 21, No. 24, pp. 7519-7541, 2017.

[24] R. Cheng and Y. Jin, "A competitive swarm optimizer for large scale optimization", IEEE Transactions on Cybernetics, Vol. 45, No. 2, pp.191-204, 2014.

[25] Y. Ling, Y. Zhou, and Q. Luo, "Lévy flight trajectory-based whale optimization algorithm for global optimization", IEEE Access, Vol. 5, pp. 6168-6186, 2017.

[26] S. Li, W. Gong, X. Yan, C. Hu, D. Bai, and L. Wang, "Parameter estimation of photovoltaic models with memetic adaptive differential evolution", Solar Energy, Vol. 190, pp. 465-474, 2019.

[27] X. Chen and K. Yu, "Hybridizing cuckoo search algorithm with biogeography-based optimization for estimating photovoltaic model parameters", Solar Energy, Vol. 180, pp. 192 206, 2019.

[28] X. Chen, B. Xu, C. Mei, Y. Ding, and K. Li, "Teaching-learning-based artificial bee colony for solar photovoltaic parameter estimation", Applied Energy, Vol. 212, pp. 1578-1588, 2018.

[29] K. Yu, J. J. Liang, B. Y. Qu, Z. Cheng, and H. Wang, "Multiple learning backtracking search algorithm for estimating parameters of 
photovoltaic models", Applied Energy, Vol. 226, pp. 408-422, 2018.

[30] D. Oliva, M. A. El Aziz, and A. E. Hassanien, "Parameter estimation of photovoltaic cells using an improved chaotic whale optimization algorithm", Applied energy, Vol. 200, pp. 141$154,2017$.

[31] H. Chen, S. Jiao, A. A. Heidari, M. Wang, X. Chen, and X. Zhao, "An opposition-based sine cosine approach with local search for parameter estimation of photovoltaic models", Energy Conversion and Management, Vol. 195, pp. 927-942, 2019.

[32] G. Xiong, J. Zhang, X. Yuan, D. Shi, Y. He, and G. Yao, "Parameter extraction of solar photovoltaic models by means of a hybrid differential evolution with whale optimization algorithm," Solar Energy, Vol. 176, pp. 742761, 2018.

[33] W. Long, S. Cai, J. Jiao, M. Xu, and T. Wu, “A new hybrid algorithm based on grey wolf optimizer and cuckoo search for parameter extraction of solar photovoltaic models", Energy Conversion and Management, Vol. 203, pp. 112-243, 2020.

[34] A. Valdivia-González, D. Zaldívar, E. Cuevas, M. Pérez-Cisneros, F. Fausto, and A. González, "A chaos-embedded gravitational search algorithm for the identification of electrical parameters of photovoltaic cells", Energies, Vol. 10, No. 7, pp. 1052, 2017.

[35] G. G. Wang, L. Guo, A. H. Gandomi, G. S. Hao, and H. Wang, "Chaotic krill herd algorithm", Information Sciences, Vol. 274, pp. 17-34, 2014.

[36] M. Kohli and S. Arora, "Chaotic grey wolf optimization algorithm for constrained optimization problems", Journal of Computational Design and Engineering, Vol. 5, No. 4, pp. 458-472, 2018.

[37] S. Arora and P. Anand, "Chaotic grasshopper optimization algorithm for global optimization", Neural Computing and Applications, Vol. 31, No. 8, pp. 4385-4405, 2019.

[38] A. H. Gandomi, X.-S. Yang, S. Talatahari, and A. H. Alavi, "Firefly algorithm with chaos", Communications in Nonlinear Science and Numerical Simulation, Vol. 18, No. 1, pp. 89-98, 2013.

[39] B. Wu and S. Fan, "Improved artificial bee colony algorithm with chaos", in International Workshop on Computer Science for Environmental Engineering and EcoInformatics, Springer, pp. 51-56, 2011.

[40] G. Kaur and S. Arora, "Chaotic whale optimization algorithm", Journal of
Computational Design and Engineering, Vol. 5, No. 3, pp. 275-284, 2018.

[41] A. H. Gandomi and X.-S. Yang, "Chaotic bat algorithm", Journal of Computational Science, Vol. 5, No.2, pp. 224-232, 2014. 